\title{
Structural and Acoustic Damping Characteristics of Polyimide Microspheres
}

\author{
Daniel L. Palumbo \\ NASA Langley Research Center, Hampton, VA 23606 \\ Junhong Park ${ }^{\dagger}$ \\ Hanyang University, Seoul, South Korea
}

\begin{abstract}
A broad range of tests have been performed to evaluate the capability of tiny lightweight polyimide spheres to reduce sound and vibration. The types of testing includes impedance tube measurement of propagation constant, sound power insertion loss for single and double wall systems, particle frame wave characterization and beam vibration reduction. The tests were performed using spheres made of two types of polyimide and with varying diameter. Baseline results were established using common noise reduction treatment materials such as fiberglass and foam. The spheres were difficult to test due to their inherent mobility. Most tests required some adaptation to contain the spheres. One test returned obvious non-linear behavior, a result which has come to be expected for treatments of this type. The polyimide spheres are found to be a competent treatment for both sound and vibration energy with the reservation that more work needs to be done to better characterize the non-linear behavior.
\end{abstract}

\section{Introduction}

Polyimide microspheres made from Teek polymer are a precursor to a robust foam that is being considered for use in several aerospace applications ${ }^{1}$. The tiny $(400-1200 \mu \mathrm{m})$ spheres were studied to better understand their potential as a lightweight noise and vibration treatment. The use of particle damping systems has been a subject of continued interest (see $\mathrm{Mao}^{2}$ for a recent overview) with progress being made in modeling the behavior of particle systems. The focus here is more on the bulk behavior of the spheres with tests designed to measure the material's acoustic properties. Due to the nature of the material, i.e., much like a foam, the particles support acoustic waves through both the fluid medium (air) which surrounds the particles and the particle's polymer frame.

Four different tests have been performed on the material, each designed to return data on specific acoustic properties. Impedance tube tests were done to obtain the material's propagation constant whose real part is the absorption coefficient and imaginary part is the wave number. Although the wave speed can be derived from the wave number, the nature of the impedance tube test is such that it is difficult to know what type of wave (fluid or frame) dominates the result. The second set of tests measures the material's sound power insertion loss, i.e., its ability to reduce transmitted vibration and radiated sound. While this test looks primarily at the radiated wave in the fluid (air), the effect of Lodengraf damping ${ }^{3}$, a structural damping phenomenon, can be observed with its effect on radiated sound power. The third test is targeted specifically at characterizing the material's dynamic frame properties. This test (and the fourth test) uses the transfer function method as described by Park ${ }^{4}$ to derive the dynamic properties of the material over the frequency band of interest. This is useful in that common techniques can return damping factors only at modal frequencies, thus obscuring the broadband characteristics of the material. In this test the non-linearity of the material becomes evident. The fourth test evaluates the microspheres as a structural damping treatment in beams, measuring the beam bending stiffness loss factor. Again, the broadband result allows peaks in damping to be located and the properties of the material to be better understood.

For all tests, common materials such as fiberglass and acoustic foam are also tested to provide a performance baseline. Other particulates such as Perlite and 3M glass bubbles are tested as well. Two types of the Teek polymer $(\mathrm{H}$ and $\mathrm{L}$ ) which are used to make the microspheres were tested with the spheres ranging in diameter from $400 \mu \mathrm{m}$ to $1200 \mu \mathrm{m}$. Table 1 provides a summary of the properties of the materials used in the four tests.

\footnotetext{
*Senior Aerospace Technologist, Structural Acoustics Branch, MS 463, Member AIAA

${ }^{\dagger}$ Assistant Professor, School of Mechanical Engineering, 17 Haendang-dong, Seongdong-gu
} 


\begin{tabular}{|l|l|l|l|l|l|l|l|}
\hline & Fiberglass & Foam & $\begin{array}{c}\text { Teek-H } \\
(600 \mu \mathrm{m})\end{array}$ & $\begin{array}{c}\text { Teek-L } \\
(600 \mu \mathrm{m})\end{array}$ & $\begin{array}{c}\text { Coarse } \\
\text { Perlite }\end{array}$ & $\begin{array}{c}\text { Fine } \\
\text { Perlite }\end{array}$ & $\begin{array}{c}\text { Glass } \\
\text { Spheres }\end{array}$ \\
\hline \hline density $\left(\mathrm{kg} / \mathrm{m}^{3}\right)$ & 15 & 30 & 20 & 30 & 125 & 40 & 1590 \\
\hline porosity & 0.99 & 0.9 & 0.35 & 0.35 & & & \\
\hline flow resistivity & 24 & 25 & 80 & 80 & & & \\
\hline $\begin{array}{l}\text { elastic modulus } \\
\text { (MPa @ 1KHz) }\end{array}$ & 0.0054 & 0.26 & 0.03 & 0.04 & & & \\
\hline
\end{tabular}

Table 1. Properties of Materials.

\section{Impedance Tube Measurements}

An impedance tube, such as the one pictured in Fig. 1, can be used to measure the propagation constant of the material of interest. The propagation constant, $\gamma$, is a complex quantity which combines the material's absorption coefficient, $\alpha$, as the real part with the associated wave number, $k$, as the imaginary part. Several methods exist for extracting these quantities from the experimental measurements ${ }^{5}$. Here a wave number space method ${ }^{6}$ was developed $^{2}$ to provide more flexibility in analyzing material of different thicknesses and properties.

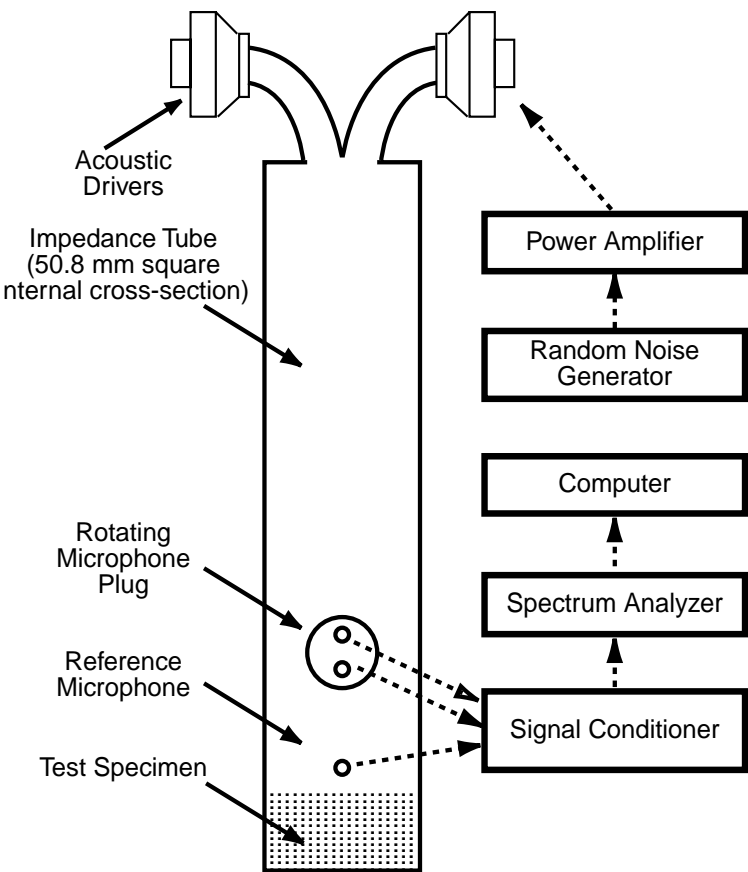

igure•1.•Diagram of Vertical Impedance Tube.

\section{A. Experimental Setup}

A sketch of NASA Langley's Vertical Impedance Tube (VIT) is shown in Fig. 1. This normal incidence impedance tube is mounted in a vertical configuration such that bulk materials can easily be tested. Most test specimens are then held in place by gravity, thus eliminating the need for a restraining layer (e.g., a wire mesh screen). In the case of the lightweight microspheres, a nylon mesh had to be applied over specimen to keep the material in place. The tube is approximately $0.7 \mathrm{~m}$ long, with a square cross-section $(51 \mathrm{~mm}$ by $51 \mathrm{~mm})$. Two $120-$ watt, phase-matched acoustic drivers generate acoustic plane waves in the tube for frequencies up to $3.0 \mathrm{kHz}$, with sound pressure levels up to $140 \mathrm{~dB}$ at the test specimen surface. The test material is installed in an enclosure designed to achieve an airtight seal, then aligned and clamped to the tube exit. A $6.4 \mathrm{~mm}$ condenser-type microphone, flush mounted in the wall $6.4 \mathrm{~mm}$ from the face of the test specimen, is used as a reference microphone to measure the sound pressure level near the face of the specimen. Two additional $6.4-\mathrm{mm}$ microphones are flush mounted in the side-wall of the VIT, 73 and $105 \mathrm{~mm}$ from the test specimen surface. As shown in Fig. 1, these microphones are mounted in a rotating plug, such that their locations can be accurately and conveniently switched. 


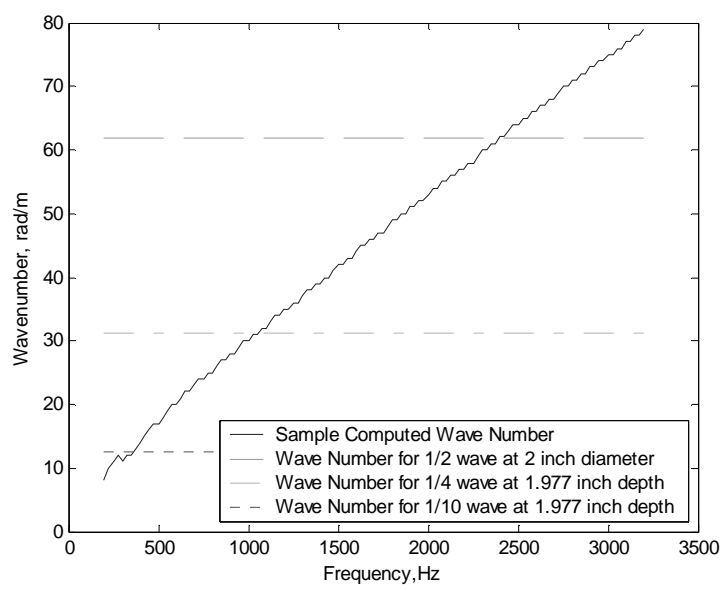

Figure $2 . \cdot$ Wave number of fiberglass measurement limits shown.

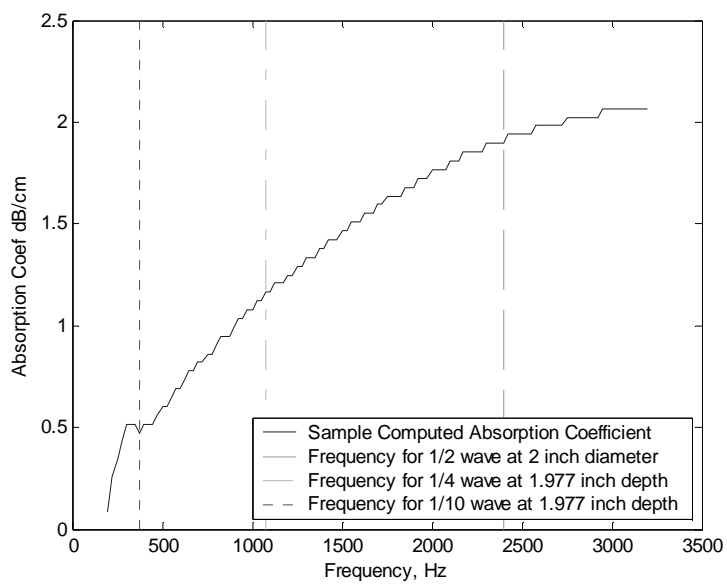

Figure 3 .'Absorption coefficient of fiberglass with measurement limits shown.

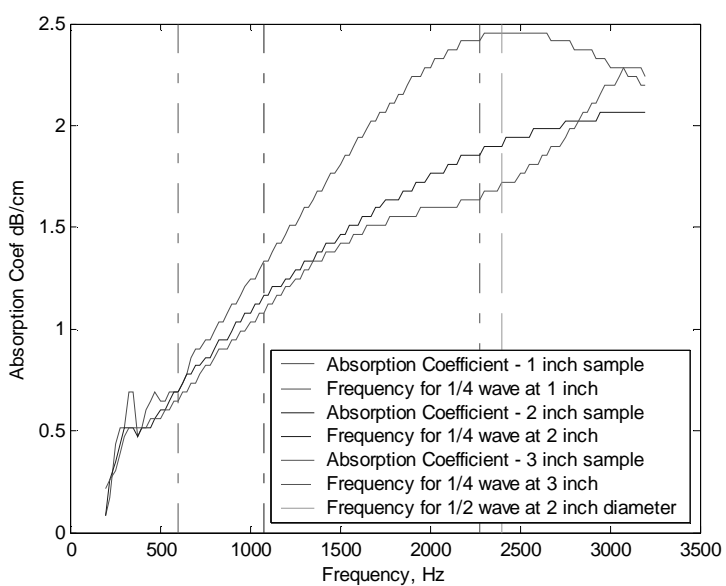

\section{B. Analysis}

For a test specimen of thickness $d_{n}$, the impedance tube measurement returns the specific impedance, $Z_{n}$. The specific impedance is related to the characteristic impedance, $\mathrm{Z}_{\mathrm{c}}$, by

$$
\mathrm{Z}_{\mathrm{n}}=\mathrm{Z}_{\mathrm{c}} \operatorname{coth}\left(\gamma d_{n}\right)
$$

If 2 different thicknesses are measured then the following solution of the simultaneous equations can be formed.

$$
0=\mathrm{Z}_{1} / \operatorname{coth}\left(\gamma d_{2}\right)-\mathrm{Z}_{2} / \operatorname{coth}\left(\gamma d_{1}\right)
$$

By evaluating Eq. 2 over the complex wave number/ frequency domain of interest, the roots (solutions) can be extracted ${ }^{6}$.

\section{Example: Fiberglass}

Fiberglass returns well behaved data from impedance tube testing. An example of the wave number and absorption coefficient properties extracted from 2 samples of fiberglass are shown in Fig. 2 and Fig. 3. Also shown in these figures are lines which denote the wave numbers (in the case of Fig. 2) and frequencies (in Fig. 3) at which certain measurement limits are reached. The green dashed line in the figures is the point at which a cross mode would turn on in the material (across the 51 $\mathrm{mm}$ tube). This is significant because this resonance may corrupt the reflected signal that is sensing the material properties. The cyan, dot-dashed line, is the point at which the 1/4 wavelength will exist in the material. This is important because at this point, velocities (and therefore absorptivity) will peak in the material. The red, dotted, line is the point at which only $1 / 10$ of a wavelength will exist in the material, corresponding to low velocities, and, therefore low absorption, resulting in a poor measurement.

The effects of these limits are illustrated in Fig. 4 where 3 analyses were done using sample pairs where the thinner sample was 1", 2" and 3". The dash-dotted lines correspond to the 1/4 wavelength point for the similarly colored data line. The green dash line is the cross mode cut-on point. For a given material, the 1/4 wavelength point varies inversely with the thickness. The 1" sample (red line) has a 1/4 wavelength point very near the cross mode cut-on frequency. This strengthens the reflected signal and reduces the effect of the cross mode. The 3" sample (green line) has its $1 / 4$ wavelength point just above $500 \mathrm{~Hz}$. The cross mode effect is very strong with the observed error in measurement. It is important to remember these measurement limitations when interpreting the data taken with the impedance tube.

Figure 4 .Effect of sample thickness on measured absorption coefficient for fiberglass. 

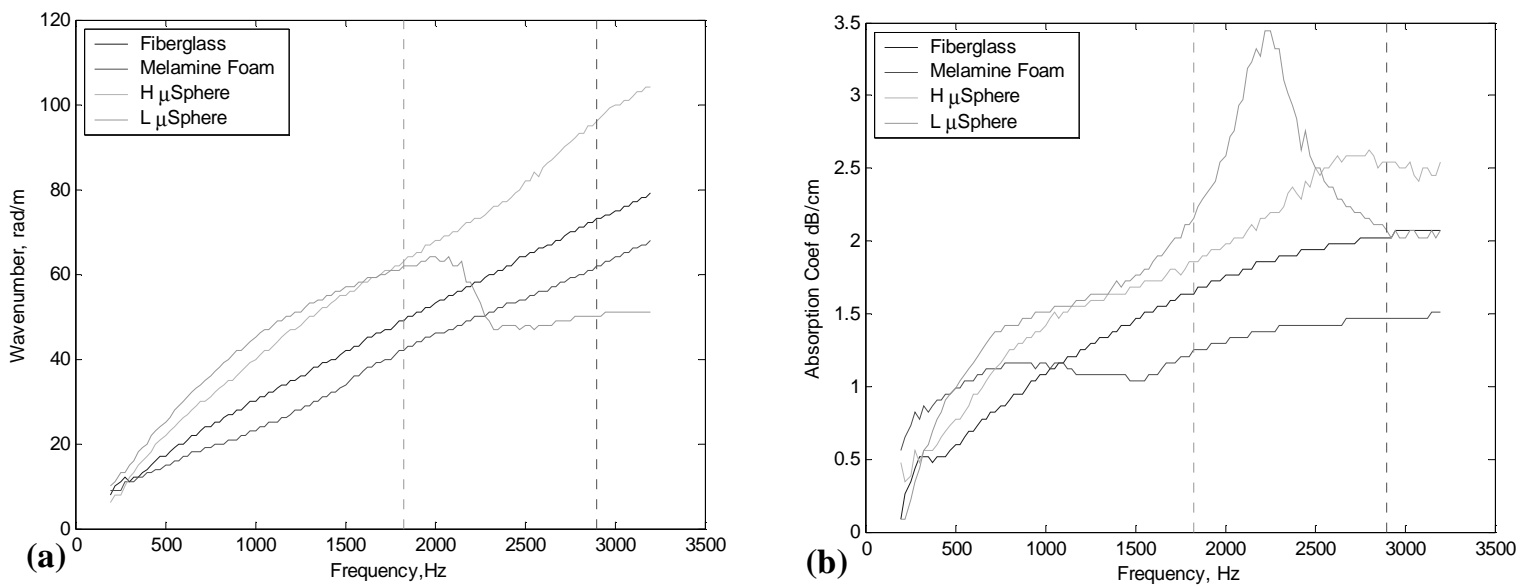

Figure $5 . \cdot$ Wave number (a) and absorption coefficient (b) for fiberglass, melamine foam, H-type and L-type microspheres with cross mode frequency limits shown

\section{Microsphere Results}

The most noticeable feature of the microsphere data is the discontinuity that occurs in the L-type results for frequencies above the cross-mode cut-on in both the wave number and absorption coefficient (Fig. 5). The crossmode limits are for the lowest and highest frequency cases, L-type microsphere and melamine foam, respectively. Before concluding that the discontinuity is due to a very strong cross mode, one has to take a careful look at the melamine foam result, especially the absorption coefficient. Note that a dip occurs in the melamine absorption coefficient around $1500 \mathrm{~Hz}$, way below the cross-mode cut-on. This case is the mildest form of this type of behavior observed in the foam with most results showing extreme discontinuities, much like the microsphere L-type.

It is suspected that this kind of behavior may be due to the two types of acoustic waves, fluid and frame, which exist in the foam, with the dominant wave behavior changing in the analysis band. The microspheres can be successfully modeled as a porous material supporting both types of waves ${ }^{4}$, leading to the expectation that a similar dual behavior would be observed. An analysis taking the two wave behaviors into account would have to be done to deconvolve the results. Note that the fiberglass does not have a significant frame wave which may be why its propagation constant is so well behaved. The data show that both types of microspheres have better absorption properties than fiberglass throughout the frequency range, and, better than the foam above $700 \mathrm{~Hz}$.

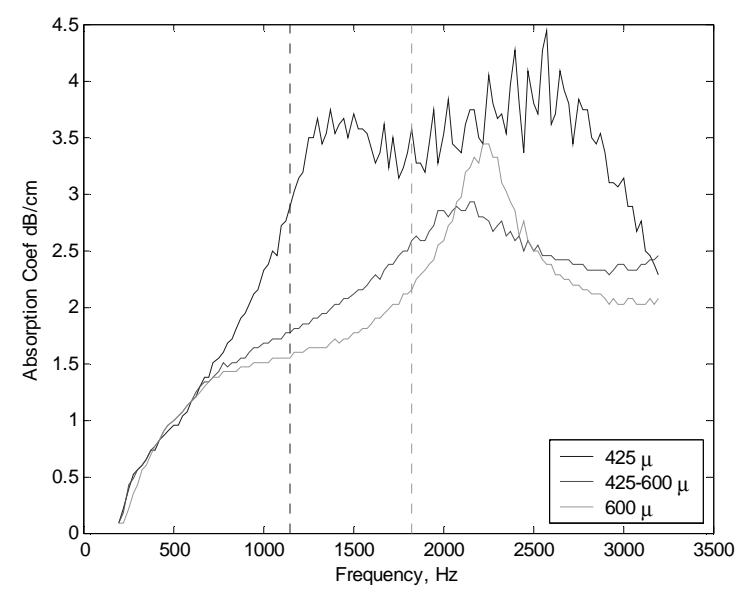

Figure $6 . \cdot$ Absorption coefficient of H-type $\mu$ Spheres of different diameters with bounds of upper measurement limits.

To obtain the effect of particle size, the H-type microspheres were sifted into groups of 3 diameter ranges; $<425 \mu \mathrm{m}, 425$ to $600 \mu \mathrm{m}$, and, >600 $\mu \mathrm{m}$ and tested. The respective absorption coefficients for these materials are shown in Fig. 6. The absorption coefficients appear identical below $\sim 700 \mathrm{~Hz}$ and then separate, with the smaller particles exhibiting greater absorption. The smaller particles have a very low cross mode frequency which may be biasing these results. However, the results do show some promise and that further testing was justified.

One of the common applications of a noise treatment is between a double wall partition such as between the exterior and interior walls of a home, or, in an airplane between the fuselage skin and the interior trim. A characteristic of the double wall system is the mass-airmass resonance frequency, i.e., the frequency at which the gap between the walls resonates due to the mass of the sidewalls and the elasticity of the air/treatment between them. The significance of this frequency is that once past this point, the transmission loss exhibits an $18 \mathrm{~dB}$ per octave gain. The mass-air-mass resonant frequency is inversely proportional to the gap distance, but, proportional to the phase speed of air (material) in the gap. Thus, a double wall with a larger gap will have a lower mass-air-mass resonant frequency. Similarly, to obtain a treatment 
with improved low frequency performance over fiberglass, it may be possible to replace the fiberglass with a material with a lower phase speed and thus a correspondingly lower resonant frequency.

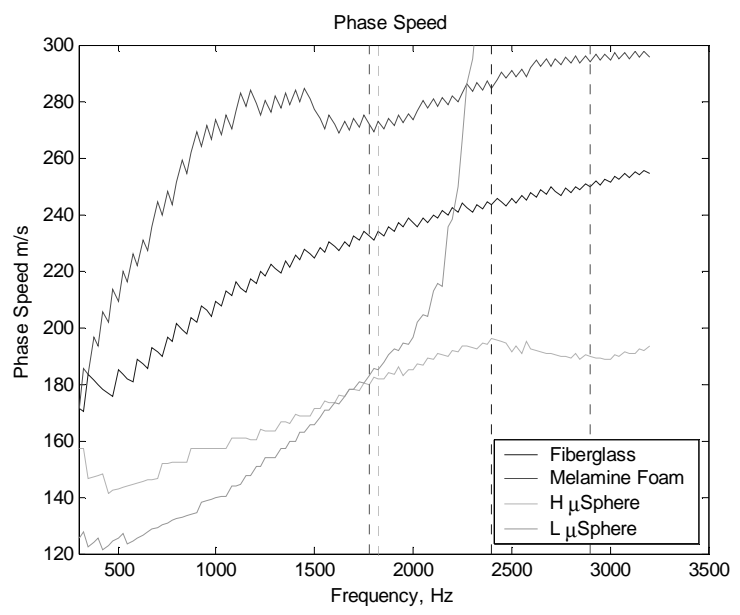

Figure $\cdot$ 7.Phase speeds of different materials with upper measurement limit for each material.

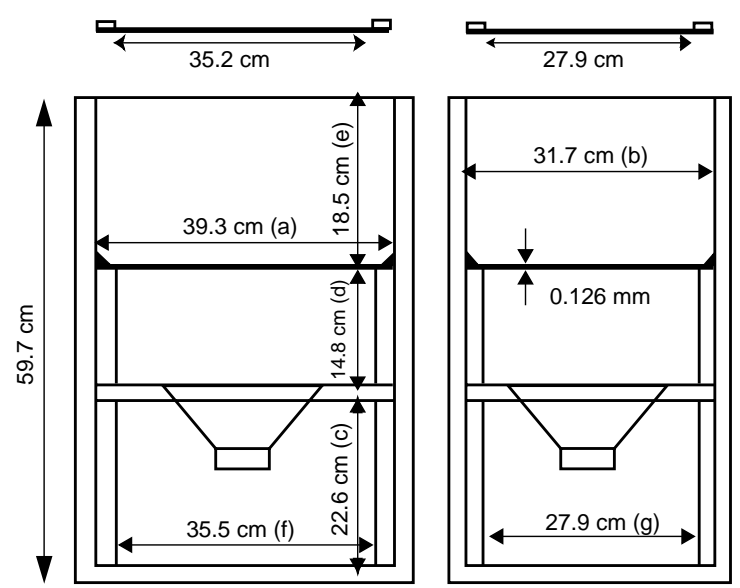

Figure 8 .・Diagram of Test Box

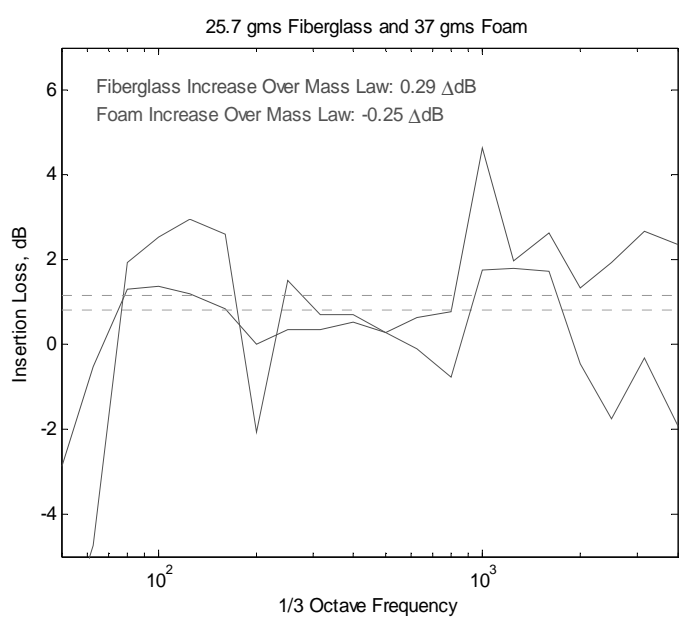

Figure $9 . \cdot$ Insertion loss of $1.27 \mathrm{~cm}$ acoustic foam and fiberglass
Material phase speed may be obtained from the wave number/frequency data. The derived phase speeds for the fiberglass, foam and microspheres are shown in Fig. 7 along with the cross mode limit frequency. As can be expected, materials with lower phase speeds have a lower cross-mode limit frequency. The microspheres have very low phase speeds compared to the foam and fiberglass and could be expected to have lower mass-air-mass resonant frequencies. As will be shown in the following section, this was not found to be the case.

\section{Insertion Loss Tests}

A reconfigurable test setup was devised such that a treatment's insertion loss could be measured when applied to a single aluminum panel and also between 2 panels in a double-walled configuration (see Fig. 8). The test box is divided into 3 chambers. The lower chamber houses an 8 in. loudspeaker that generates the source noise. The center chamber supports the first panel above the speaker. Both of these chambers are damped with fiberglass. The walls of the upper chamber provide support so that a second panel can be installed at different heights above the first panel, creating double-walled cavities of varying depths. Several types of material were tested using this setup including fiberglass, foam, perlite and polyimide microspheres ${ }^{7}$.

\section{A. Single Panel Tests}

The average radiated sound power is measured by scanning an intensity probe over the open end of the box. Insertion loss is obtained by comparing the measured sound power of the baseline, untreated, condition to the treated condition. In Fig. 9 are shown the insertion losses for fiberglass and acoustic foam for a single panel test, i.e., the treatment is placed above the first panel and the second panel is not installed. The dotted line is the insertion loss that would have been obtained if the panel's mass were increased by the mass of the treatment. An insertion loss above this line is an indication of good performance.

The expected behavior for these common materials is observed. The fiberglass shows no appreciable insertion loss below $1 \mathrm{kHz}$ and very good insertion loss above $1 \mathrm{k}$ $\mathrm{Hz}$. The foam has much better low frequency insertion loss with a reduced high frequency loss, especially above $2 \mathrm{kHz}$. Note the steep fall-off above $2 \mathrm{kHz}$ as this kind of behavior will be seen in several materials. 


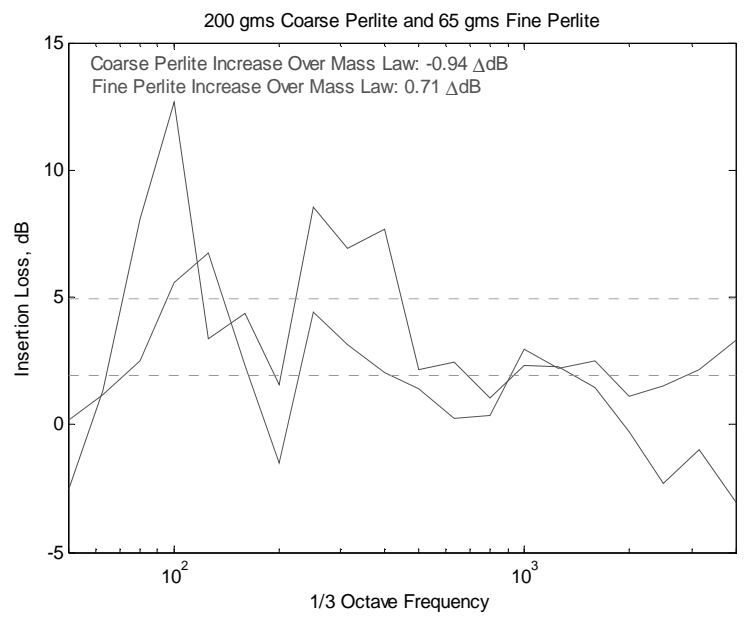

Figure $10 . \cdot$ Insertion loss of $1.27 \mathrm{~cm}$ of coarse and fine perlite.

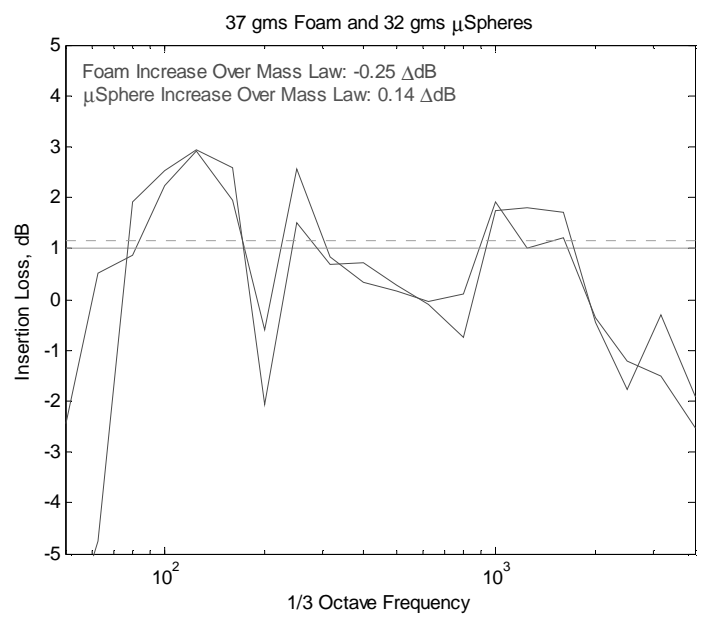

Figure•11.•Insertion loss of $\mu$ spheres on lower plate.

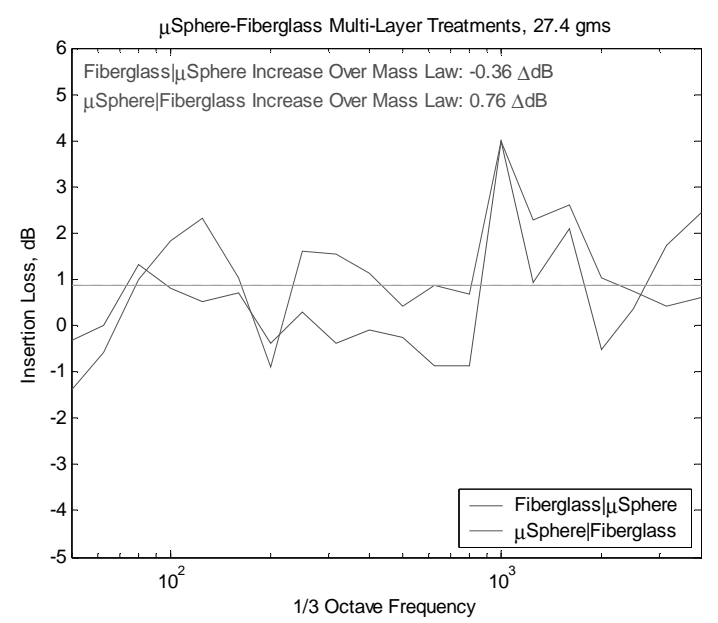

Figure 12 . Insertion Loss dependence on ordering of microspheres and fiberglass treatments
Perlite has attracted attention as a vibration and noise reduction treatment due to a behavior described as Lodengraf, or radiation, damping ${ }^{3}$. The underlaying mechanics of the behavior are due to the treatment having a slow (with respect to the speed of sound in air) phase speed so that the treated surface radiates energy more easily into the treatment. This behavior should result in increased vibration damping. However, any decrease in sound radiation will depend on how well the material dissipates the additional acoustic energy it has absorbed.

The frequency at which Lodengraf damping can be expected to take effect can be calculated as the frequency at which the speed of sound in the panel is equal to the speed of sound in the treatment, or the critical frequency ${ }^{8}, f_{c}$.

$$
f_{c}=c^{2} / 1.8 h c_{l}
$$

where $c$ is the speed of sound in the treatment, $h$ is the plate thickness and $c_{l}$ is the longitudinal wave speed in the plate. For the aluminum plate used in this test, the nominal critical frequency with respect to air would be around $13 \mathrm{KHz}$. Two types of Perlite were tested, common, or coarse, and very fine. The respective insertion losses are shown in Fig. 10. The coarse perlite, with a published wave speed of $62 \mathrm{~m} / \mathrm{s}$, has a computed critical frequency of $428 \mathrm{~Hz}$, in excellent agreement with the observed falloff in insertion loss. The fine perlite does not show a similar behavior. Due to the increased coupling of vibration energy into the perlite, the coarse Perlite returns a negative insertion loss.

The microspheres have a phase speed approximately double that of the perlite, $\sim 140 \mathrm{~m} / \mathrm{s}$ at $1 \mathrm{KHz}$. This would put the critical frequency at about $1600 \mathrm{~Hz}$ where a steep drop in insertion is observed, Fig. 11. The microsphere insertion loss is seen to be very similar to foam, suggesting that the acoustic foam may have Lodengraf properties as well.

When comparing the microspheres performance to the fiberglass it is seen that the fiberglass has better high frequency performance while the microspheres have better low frequency performance. A test was performed to see if a multi-layer treatment could achieve good performance across the analysis band. It was observed, Fig. 12, that the multi-layer treatment was order dependent, i.e., the multi-layer treatment worked better when the microspheres were in contact with the lower plate. The low frequency damping of the microspheres was maintained and the high frequency fall-off was compensated by the fiberglass. These somewhat surprising results were confirmed in an analytical study as will be shown later. 


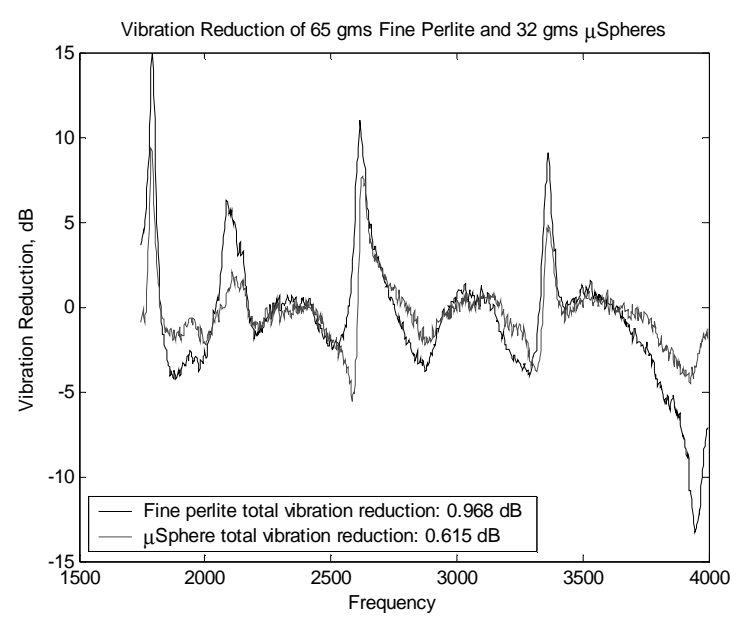

Figure $\bullet 13 . \bullet$ Vibration reduction of lower plate with fine perlite and microsphere treatments.

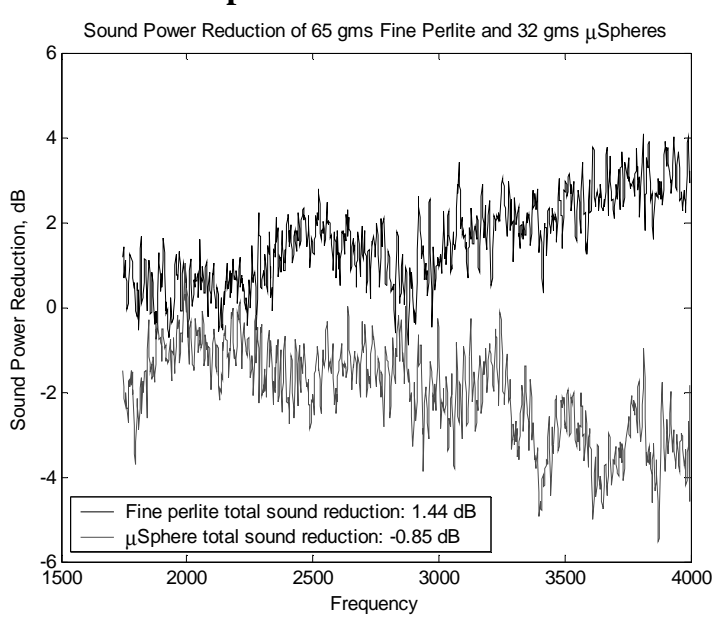

Figure $14 . \bullet$ Average change in sound intensity with and without fine perlite and microsphere treatments.

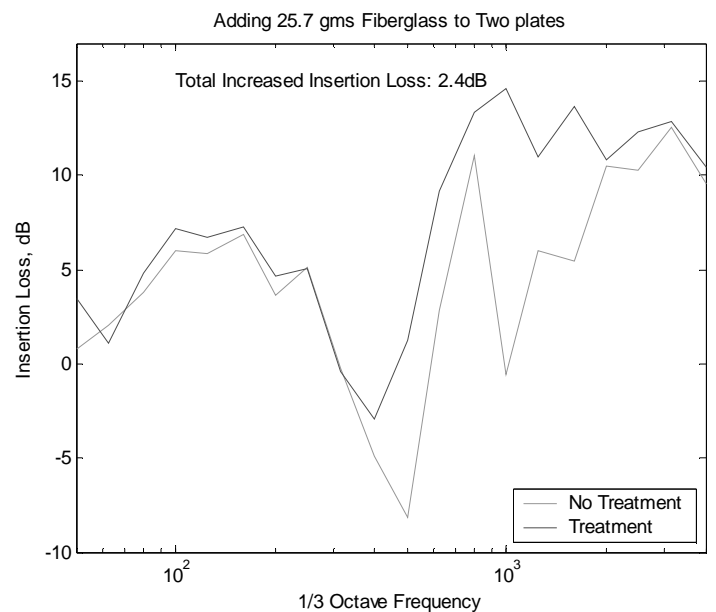

Figure $15 . \cdot D o u b l e$ plate insertion loss with and without fiberglass.

\section{B. Evidence of Lodengraf Damping}

A key signature of Lodengraf damping would be a reduction in vibration of the structure with an accompanying increase in the sound power radiation. To test if this is indeed the case, the fine grain Perlite is compared to the microspheres in the frequency range of 1500 to $4000 \mathrm{~Hz}$ where it is assumed that the microspheres are exhibiting Lodengraf effects. As the coarse Perlite is known to produce Lodengraf behavior it would have been preferred except that its very high mass would have skewed the results. The added masses of the fine Perlite and microspheres (at 65 and 32 gms respectively) are close enough to allow a comparison.

The change in vibration response caused by the application of the material to the plate was measured by an accelerometer placed beneath the plate. The vibration reduction is plotted in Fig. 13. The fine Perlite has a total vibration reduction of $0.97 \mathrm{~dB}$ and the microspheres 0.62 $\mathrm{dB}$.

Given a reduction in vibration, one would expect a reduction in radiated sound power. This is exactly what is observed for the fine Perlite in Fig. 14 where the radiate sound power reduction is plotted in the analysis band. Notice, however, that the microspheres produce an increase in radiated sound power, the signature evidence of Lodengraf damping. The added mass of the fine Perlite reduces panel vibration which causes a reduction in radiated sound power. The added mass of the microspheres will also reduce panel vibration, but their improvement to the panel's radiation efficiency causes an increase in radiated sound power.

\section{Double Panel Tests}

As mentioned earlier, it was postulated that due to phase speeds lower than those of either foam or fiberglass, that the microspheres should yield very good results in a double wall configuration. An example of how fiberglass behaves in the double wall configuration is shown in Fig. 15. The no-treatment case (empty cavity) is plotted as the green line. The system exhibits a clear mass-air-mass resonance at $500 \mathrm{~Hz}$ followed by a steep $18 \mathrm{~dB} /$ octave increase in insertion loss, classic double wall behavior ${ }^{8}$. A second acoustic resonance is seen around $1000 \mathrm{~Hz}$.

When fiberglass is added (red line in Fig. 15), not only are all the resonances damped, but the apparent mass-airmass resonant frequency has dropped to $400 \mathrm{~Hz}$. An expected behavior due, ostensibly, to the lower phase speed in fiberglass. 


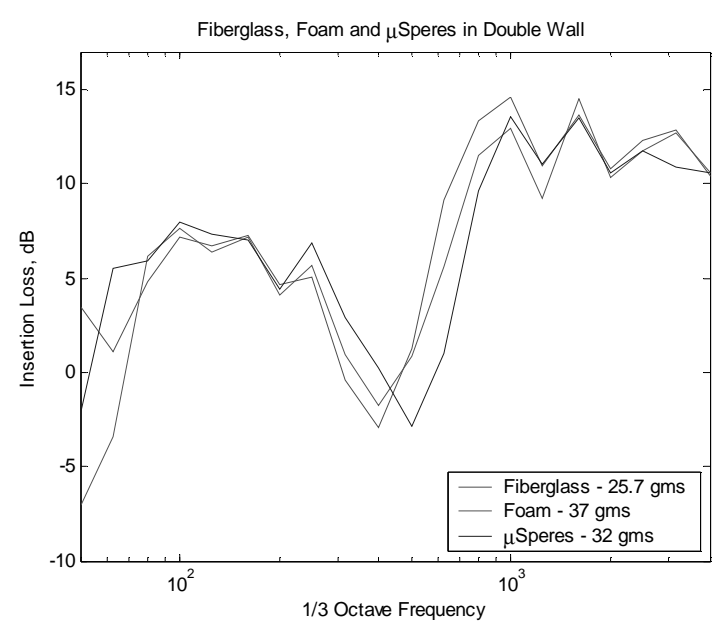

Figure $16 . \cdot D o u b l e$ wall performance of fiberglass, foam and microspheres compared.

\begin{tabular}{|l|c|c|}
\hline \multicolumn{1}{|c|}{ Treatment Type } & \multicolumn{2}{c|}{$\begin{array}{c}\text { Noise Reduction } \\
\text { dB/\% added mass }\end{array}$} \\
\hline \hline & Single Plate & Double Plate \\
\hline$\mu$ sphere @ $1.3 \mathrm{~cm}$ & $0.14 / 12 \%$ & $4.0 / 6 \%$ \\
\hline fiberglass @ $1.3 \mathrm{~cm}$ & $0.29 / 10 \%$ & $2.4 / 5 \%$ \\
\hline foam @ $1.3 \mathrm{~cm}$ & $-0.25 / 14 \%$ & $3.3 / 5 \%$ \\
\hline coarse perlite @ $1.3 \mathrm{~cm}$ & $-0.90 / 77 \%$ & $2.1 / 38 \%$ \\
\hline fine perlite @ $1.3 \mathrm{~cm}$ & $0.71 / 25 \%$ & $4.4 / 13 \%$ \\
\hline f_glass/ $\mu \mathrm{sphere@} 1.3 \mathrm{~cm}$ & $-0.36 / 11 \%$ & $1.6 / 5.3 \%$ \\
\hline$\mu$ sphere/f_glass @ $1.3 \mathrm{~cm}$ & $0.76 / 11 \%$ & $2.2 / 5.3 \%$ \\
\hline
\end{tabular}

Table 2. Test Matrix

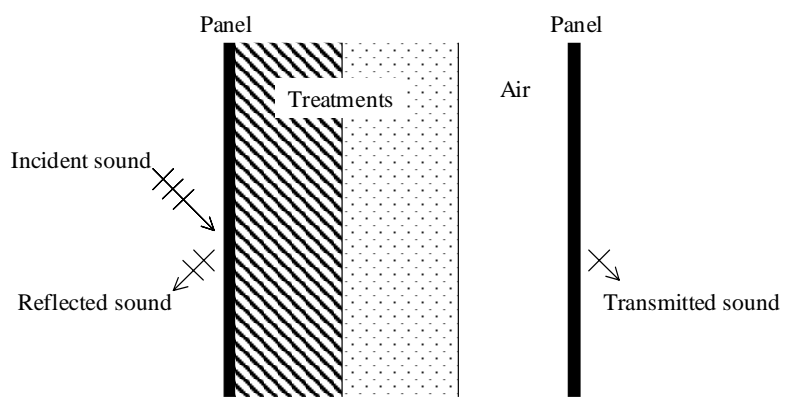

Figure $\bullet 17 . \bullet$ Multi-layer treatment model
When the double wall cavity is treated with microspheres, good sound reduction is obtained, but the expected shift in mass-air-mass resonance is not observed, Fig. 16. Here the microsphere insertion loss (blue) is plotted with that of fiberglass (red) and foam (green). Note that all these materials exhibit similar damping behavior, but that only the fiberglass and foam lower the mass-air-mass resonant frequency. The microspheres actually increase the resonant frequency slightly. This can be explained if one considers the very low porosity of the microspheres, Table 1 , of 0.35 compared to $>0.9$ for foam and fiberglass. A low porosity would inhibit a fluid wave and force all transmitted energy into a frame wave. This greatly changes the dynamics of the double wall cavity in that the elasticity of the microspheres frame is much different than that of air. The insight that most, if not all, of the acoustic energy flows through the microsphere frame also changes how one would use the material in noise reduction applications. The frame wave characteristics of the microspheres are studied in subsequent sections.

\section{Insertion Loss Summary}

The insertion loss performance of the tested materials is summarized in Table 2. Here it can be seen that the microspheres are as lightweight as foam and fiberglass and provide the best double wall reductions. Single panel results are less than that of fiberglass due to Lodengraf radiation.

The microsphere/fiberglass combination provides the best single panel reductions, but, this performance does not carry over to the double wall. Note that these tests were all carried out with horizontal plates where that particulate could be easily positioned. Designing an application for vertical treatments would require some type of casing which would most likely affect the results.

\section{Analytical Study of Multi-Layered Treatment}

Given the measured and derived properties of the polyimide microspheres (Table 1), it is possible to model the material's behavior using Biot's theory of propagation through poro-elastic media ${ }^{9}$. The model test setup is shown in Fig. 17 as consisting of two panels in a double wall configuration with two layers of material with an air gap between the material and the transmitting panel. 


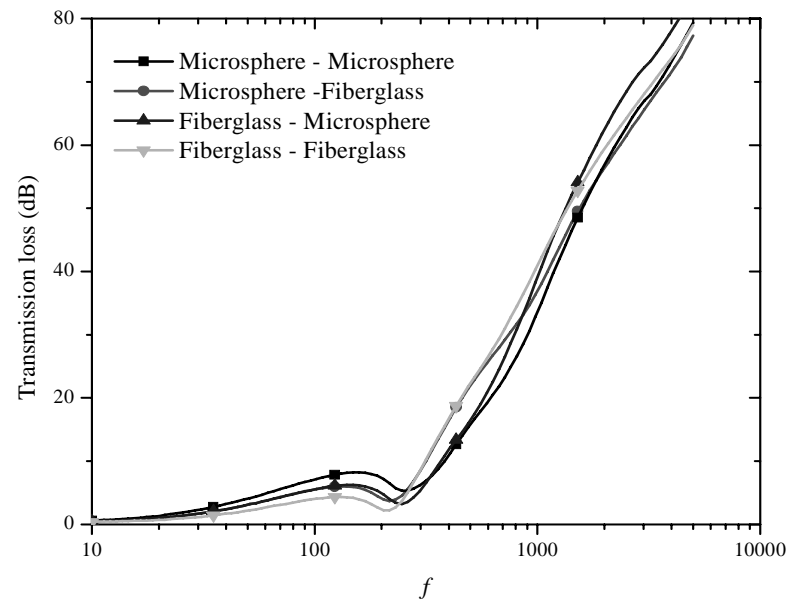

Figure $\bullet 18 . \cdot$ Transmission loss of multi-layer treatments

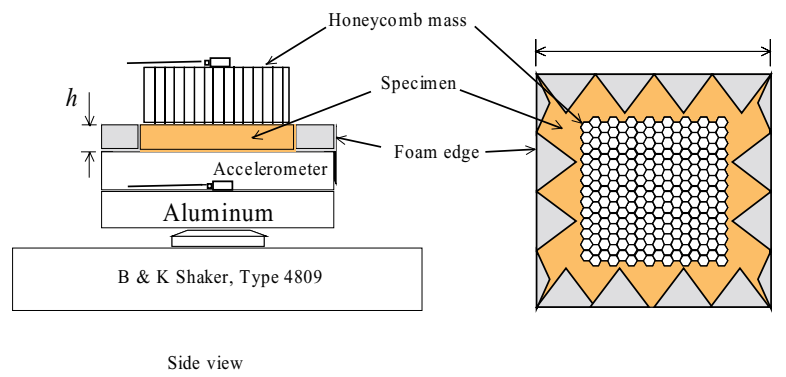

Figure•19.•Experimental setup for measuring the dynamic properties of granular material.
The most interesting result of this work was the confirmation of the experimentally observed preference in ordering of the microsphere/fiberglass combination. Fig. 18 shows the computed transmission loss for 4 combinations of microspheres and fiberglass. The black curve is the microsphere only result and exhibits better low frequency performance than the fiberglass only (cyan curve). Below the mass-air-mass resonance, the combination microsphere/fiberglass treatments lie on top of one another (red, blue curves). Above the mass-airmass resonance, though, the curves separate with the microsphere/fiberglass treatment having higher transmission loss than the fiberglass/microsphere treatment up to $800 \mathrm{~Hz}$ where the curves cross and then diverge. This result validates the experimentally observed behavior of improved performance when the microspheres are placed against the source panel. Note also that the fiberglass/microsphere combination exceeds the fiberglass transmission loss above $2000 \mathrm{~Hz}$.

\section{Particle Frame Dynamic Properties}

The measurement of the dynamic properties of a particulate material's frame is reduced to a onedimensional problem using the experiment setup shown in Fig. 19. Here the granular material is contained in a serrated foam frame which attenuates lateral vibration so that the problem reduces to the single longitudinal direction. A section of Nomex honeycomb is placed on the material to hold the particles in position. Accelerometers placed on the base of the shaker and on the honeycomb record the respective motions. Although designed primarily for granular material (here polyimide and glass microspheres), this test setup was also used to compare results to more common materials, such as foam and fiberglass. See Park ${ }^{4}$ for more details.

\section{A. Non-Linear Behavior}

The predominant feature of the microsphere transfer function data is the apparent non-linearity. In Fig. 20a are plotted the transfer functions for microspheres and acoustic foam at 5 levels of input force. The responses of the acoustic foam at the different forces overlay each other as one would expect. However, the microspheres exhibit peak broadening and a shift to lower frequencies as the input force is increased.
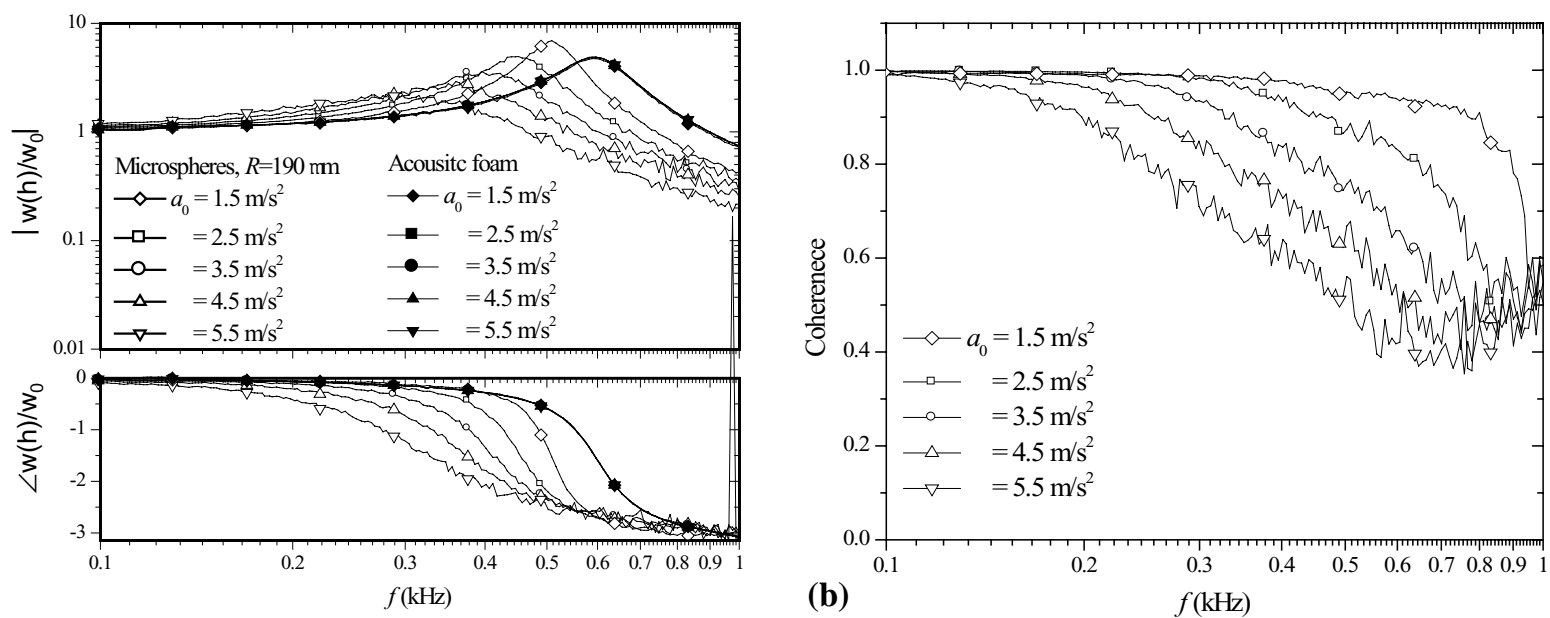

Figure•20.-Measured transfer function for microsphere $(\mathrm{R}=190 \mu \mathrm{m})$ and acoustic foam, (a) and coherence for microspheres, (b). 

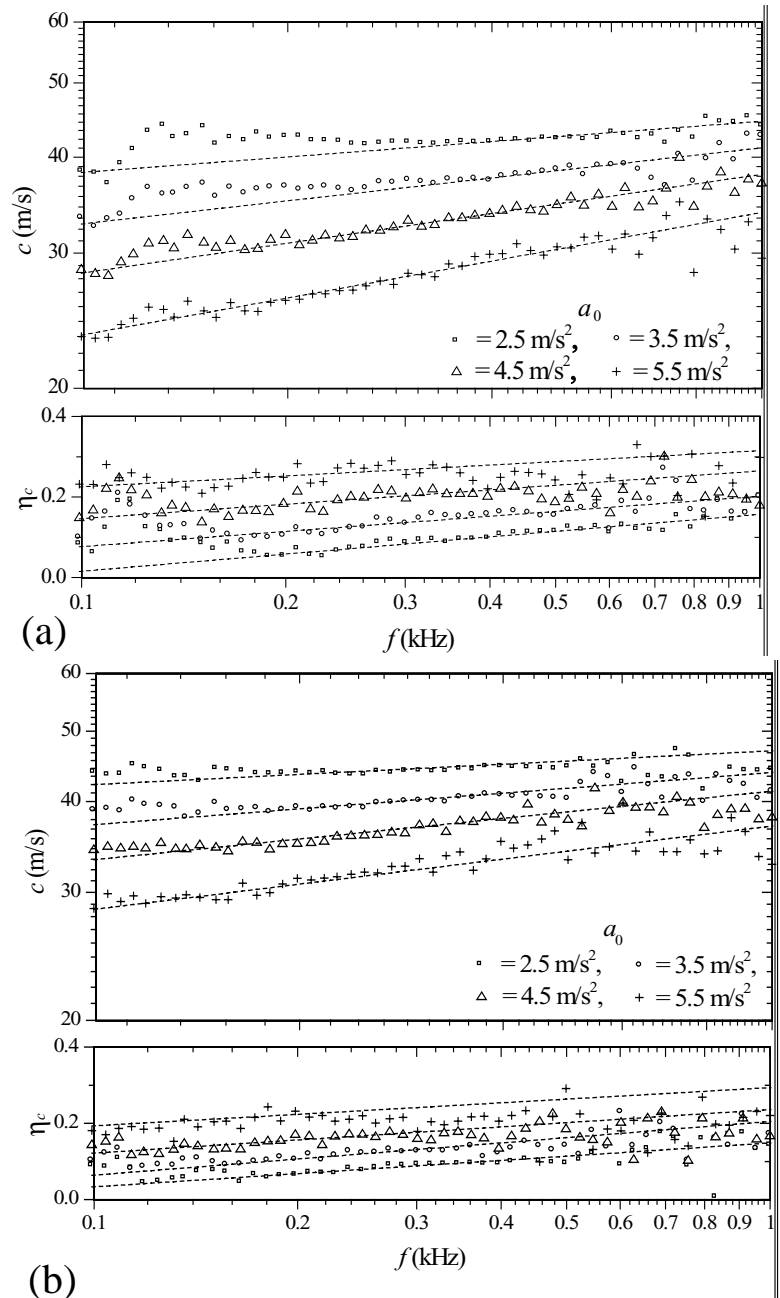

Figure $21 . \cdot$ Effect of excitation amplitude on wave speed and loss factor for microspheres, $R=190 \mu \mathrm{m}$ (a) and $\mathbf{R}=\mathbf{3 0 0} \mu \mathrm{m}$ (b).
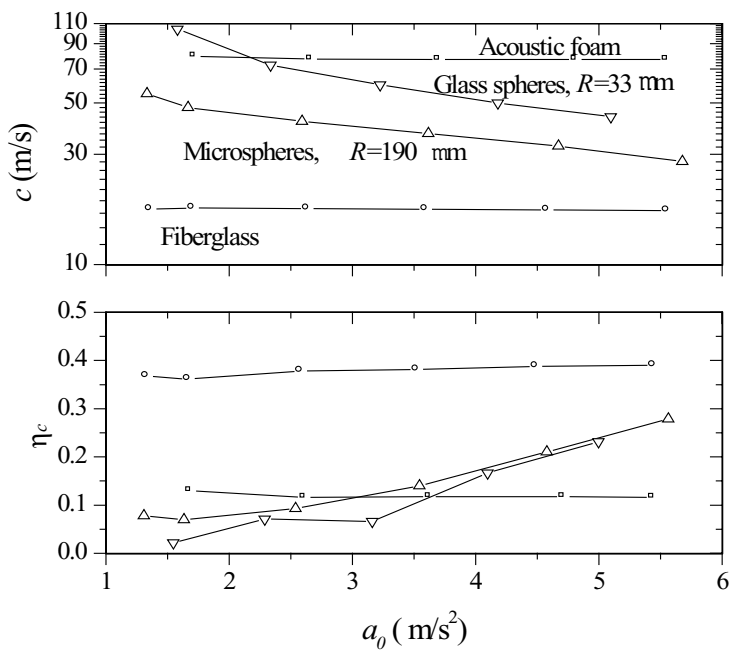

Figure $22 . \cdot W a v e$ speed and loss factor dependence on excitation amplitude for glass spheres $(700 \mathrm{~Hz})$ and for other specimens $(300 \mathrm{~Hz})$.
The coherence data (Fig. 20b) shows a corresponding drop in coherent energy with increased force. Note that the following analysis uses the linear transfer functions and are thus limited in scope to the coherent energy.

\section{B. Dynamic Analysis}

The material's wave number, $\mathrm{k}$, can be derived from the displacement transfer functions using the following relationship,

$$
\hat{\mathrm{w}}(\mathrm{x}=\mathrm{h}) / \mathrm{w}_{0}=\left(\cos \hat{\mathrm{k} h}-\left(\mathrm{m} / \mathrm{m}_{\mathrm{s}}\right) \hat{\mathrm{k} h} \sin \hat{\mathrm{k} h}\right)^{-1}
$$

where $\mathrm{w}$ is the displacement, $\mathrm{m}_{\mathrm{s}}=\rho \mathrm{Ah}$ is the mass of the specimen, $\mathrm{A}$ is the cross-section and $\mathrm{m}$ is the mass of the attached mass (the honeycomb). The expression in Eq. 4 is fit to the measured transfer function data using the Newton-Raphson method. The complex wave speed and dynamic stiffness can be derived from the wave number as described by Park ${ }^{4}$.

\section{Detail of Microsphere Non-linear Behavior}

The wave speed and its damping factor for two diameters of microspheres are shown in Fig. 21. As can be seen, the wave speed increases with frequency and decreases with applied force while the damping factor increases with frequency and applied force. Although the smaller diameter microspheres appear to have a lower wave speed and marginally higher damping factor as compared to the larger spheres, this behavior will be inverted when a wider range of diameters are analyzed in a later section. Note that sphere diameter does not affect particle mass as all the spheres are grown from the same seeds. The overall density of groups of larger spheres will then be smaller as fewer spheres will occupy the same volume. While it may appear that the smaller spheres have a greater wave speed dependence on applied force, the $y$-axis is log scale and therefore the lower wave speeds of the smaller spheres are more spread out.

These results should be understood in the context that they are, in effect, linear dynamics extracted from a nonlinear process. It might be argued that the trend in wave speed could be viewed as a dependable measurement since it is based on identifying the wave number components in the response. However, the trend in damping may be biased as a large amount of incoherent energy is unaccounted for.

\section{Linearity of Tested Materials}

The granular materials exhibit similar dynamic behavior with respect to level of excitation as shown in Fig. 22 where the wave speeds and loss factors of glass and polyimide microspheres of radius $33 \mu \mathrm{m}$ and 190 $\mu \mathrm{m}$ respectively are plotted along with acoustic foam and fiberglass. Both the foam and fiberglass have wave speeds and loss factors that are constant with applied force. The wave speeds of the granular materials decrease with applied force while the loss factors increase. Again, it is unclear if this increase in loss factor is due to an actual increase in damping or a decrease in coherent energy. 


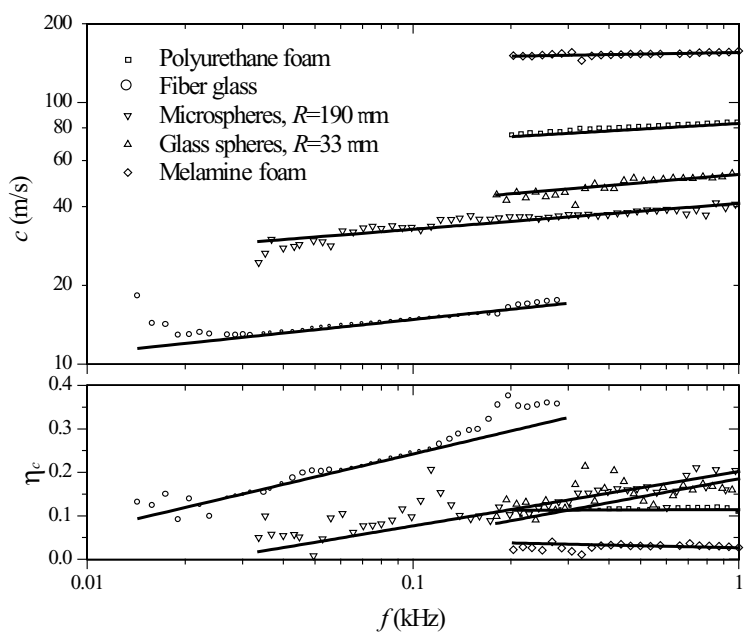

Figure $23 . \cdot$ Wave speed and loss factor for foams, fiberglass, polyimide and glass spheres at $a=3.5 \mathrm{~m} / \mathrm{sec}^{2}$
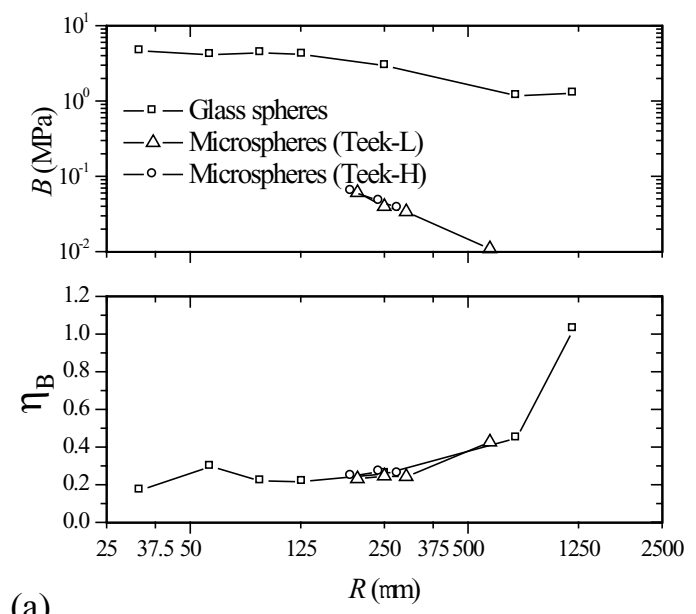

(a)
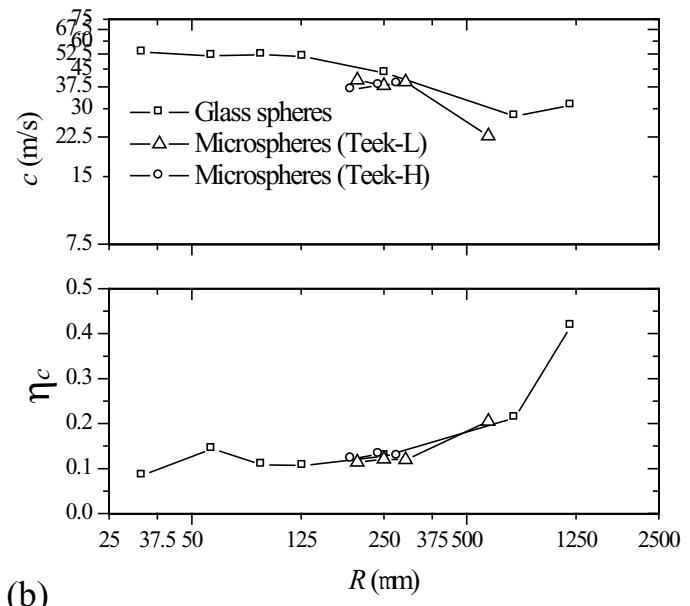

(b)

Figure $24 . \cdot$ Effect of granule size on dynamic moduli

(a) and wave speed (b), $\mathrm{a}=3.5 \mathrm{~m} / \mathrm{sec}^{2}$

\section{Comparison of Material Dynamic Properties}

Wave speeds and damping factors for the five types of materials tested are shown in Fig. 23 for an excitation level corresponding to $3.5 \mathrm{~m} / \mathrm{s}^{2}$. The foams have the highest wave speeds, followed by the glass spheres, polyimide spheres and fiberglass with the lowest wave speed. This result might appear to contradict the wave speeds obtained from the impedance tube tests (Fig. 7), but one should remember that this test measures the frame wave speed exclusively while the impedance tube will measure the total acoustic response of the material. The fiberglass has the lowest frame wave speed due to the limp nature of the fibers. The damping factor for fiberglass is also very high suggesting that the frame wave will be quickly damped. This is in contrast to the melamine foam with a very low frame damping factor indicating excellent transmission of the frame wave.

\section{Effect of Particle Size}

The granular materials, glass spheres and two types of polyimide spheres $(\mathrm{L}$ and $\mathrm{H})$, were analyzed with respect to particle diameter, Fig. 24. The glass spheres were available in a much broader range of diameters $(50-2500$ $\mu \mathrm{m})$ than the polyimide spheres $(400-1200 \mu \mathrm{m})$. The dependence of both the complex dynamic stiffness, B, and wave speed, c, on particle diameter is derived. The glass spheres are seen to be much stiffer and exhibit much less reduction in stiffness with respect to increase of particle diameter than the polyimide spheres which exhibit an order of magnitude reduction in stiffness for a factor of 3 times increase in diameter. The type of polymer does not seem to affect the measured frame stiffness of the polyimide spheres.

The damping factors of the dynamic stiffness, $\eta_{B}$, for all types of particles increase with diameter and are very close to each other. Notice that the damping factor derived from the dynamic stiffness, Fig. 24a, is identical to that derived from the wave speed, Fig. 24b.

The dependence of the materials' wave speeds on particle diameter is shown to be similar to the dynamic stiffness, i.e., the wave speeds decrease with increasing diameter. It can be seen in Fig. 24b that the wave speeds of the polyimide spheres are somewhat constant and marginally increasing in the range of $190-300 \mu \mathrm{m}$ (radius) which is in line with the results shown in Fig. 21. However, the wave speed of the $600 \mu \mathrm{m}$ (radius) polyimide spheres show a distinct drop in wave speed reinforcing the trend displayed by the glass spheres. 


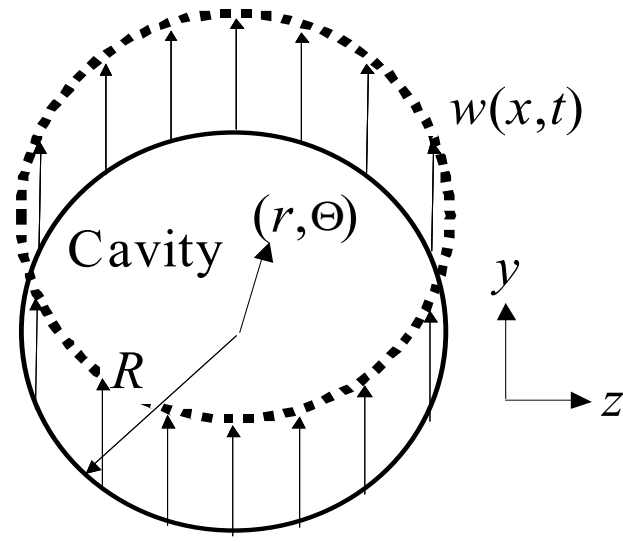

Figure $25 . \cdot$ Effect of foam and microsphere fill on transverse bending dynamics of cylindrical beams

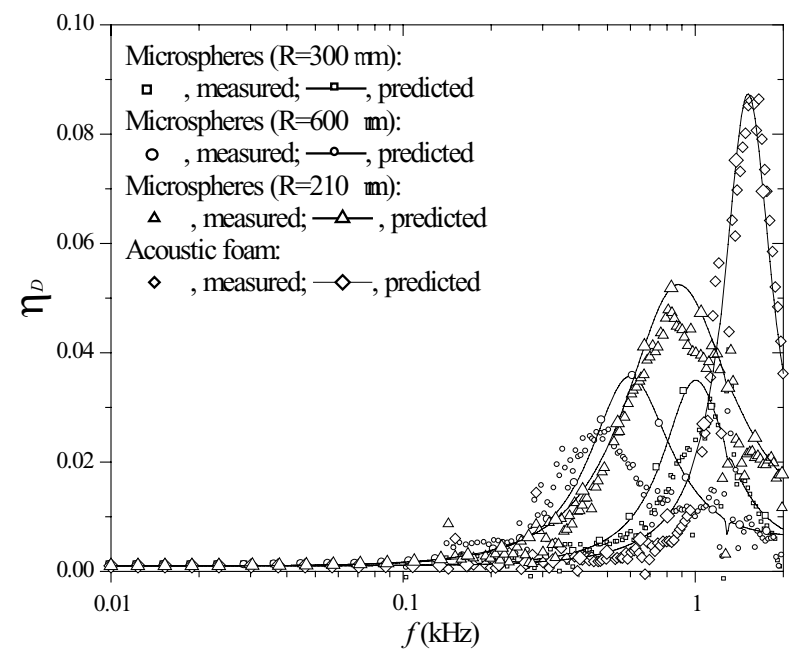

Figure $\bullet 26 . \bullet$ Measured and predicted bending stiffness loss factors for microspheres and acoustic foam.

\section{Structural Damping}

The ability of the polyimide microspheres and acoustic foam to damp transverse bending waves in a cylindrical beam has been tested using aluminum beams which were filled with the material and excited using a slip table. The aluminum beams were $0.9 \mathrm{~m}$ long with an $0.025 \mathrm{~m}$ o.d. and a $0.022 \mathrm{~m}$ i.d. The bending stiffness loss factor is derived for a continuous frequency band using the transfer function method as described by $\operatorname{Park}^{10}$. The use of the transfer function method provides damping data at off-resonant frequencies allowing the materials' maximum damping frequency to be located. The approach used here involves writing the particle/beam system's energy equations to which is then applied the Rayleigh-Ritz method (see Park ${ }^{4}$ for details). The resulting set of mass and stiffness matrices can then be used to predict the tested treatments' damping as well as the damping of a proposed treatment with given dynamic properties.

\section{A. Beam Bending Stiffness Loss factor}

Three sizes of polyimide microspheres and acoustic foam were analyzed to yield beam bending stiffness loss factor, Fig. 26. Notice how the transfer function method returns results for the loss factor for points across the analysis band and not just at modal frequencies (the first mode of the untreated beam was $30 \mathrm{~Hz}$ ). The acoustic foam returned the highest damping $(0.085)$ at the highest frequency $(>1 \mathrm{kHz})$ with good matching of measurement and predicted results. The $210 \mu \mathrm{m}$ spheres returned the next highest damping (0.05) at approximately $800 \mathrm{~Hz}$, again with a good match between measurement and prediction. The two remaining sphere sizes (600 and 300 $\mu \mathrm{m})$ returned the lowest damping peaks of about 0.025 at frequencies of 450 and $1000 \mathrm{~Hz}$ respectively. Prediction deviated from measurement for the spheres in these two cases for unknown reasons. Of interest in the results is the lower resonant peak of the larger spheres. If this behavior can be verified it may be possible to tune damping treatment to specific frequencies with particle size.
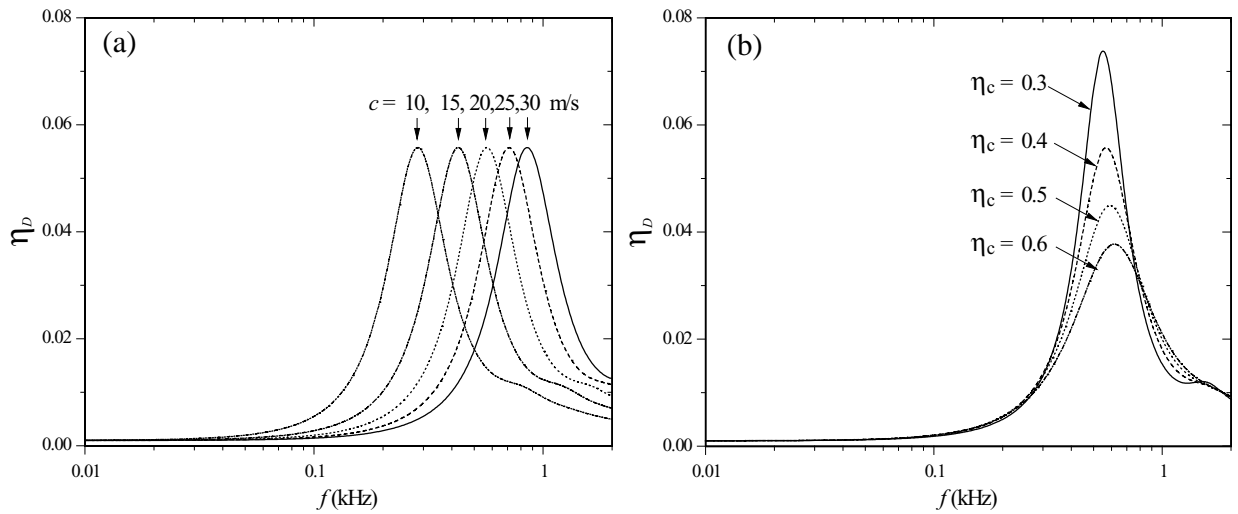

Figure 27.•Dependence of beam bending stiffness loss factor on microsphere frame wave speed for $\eta_{c}=0.4(a)$, and loss factor for $c=20 \mathrm{~m} / \mathrm{s}$ and $\rho=40 \mathrm{~kg} / \mathrm{m}^{3}$ 


\section{B. Optimal Treatment Properties}

Using the derived mass and stiffness matrices it is possible to predict the treated beam bending stiffness loss factor for treatments with different properties. Fig. 27a shows the beam bending stiffness loss factor predicted for spheres with 5 different wave speeds ranging from $10 \mathrm{~m} / \mathrm{s}$ to $30 \mathrm{~m} / \mathrm{s}$ and a constant wave speed loss factor of 0.4. It can be seen that decreasing the wave speed reduces that frequency at which the maximum beam loss factor occurs with the maximum loss factor remaining constant. Keeping the particle wave speed constant at $20 \mathrm{~m} / \mathrm{s}$ and varying the particle wave speed loss factor returns the results as shown in Fig. 27b. Here, increasing the particle loss factor reduces the maximum beam loss factor while raising the frequency at which the maximum occurs by a small amount.

\section{Conclusion}

The polyimide microspheres have been shown to be a competent, but not outstanding acoustic and vibration damping treatment. In most test cases common fiberglass and acoustic foam performed as well or better than the microspheres. In all test cases, acquiring good data was a challenge as the lightweight particles were difficult to keep in place and tended to return non-linear results. This latter effect was noted in one set of tests where the non-linearity was obvious, but may have affected other tests, for example the cylindrical beam. As an acoustic treatment the particles are a challenge to package. The cost of the material and difficulty in packaging will outweigh any marginal performance benefit over fiberglass. As a vibration treatment the microspheres offer the possibility to tune the treatment to a specific frequency depending on particle size, an effect that was observed in the data and shown to be a function of particle frame wave speed.

\section{References}

${ }^{1}$ Weiser, Grimsley, B.W., Pipes, R.B., and Williams, M.K., "Polyimide Foams from Friable Balloons", $47^{\text {th }}$ International SAMPE Symposium and Exhibition, Long Beach, CA, May, 2002, pp. 1151-1162.

${ }^{2}$ Mao, K., Wang, M.Y., Xu, Z., and Chan, T., "Simulation and Characterization of particle damping in Transient Vibrations", Transactions of the ASME, Vol. 126, April 2004, pp. 202-211.

${ }^{3}$ Fricke, J.R., "Lodengraf Damping - An Advanced Vibration Damping Technology", Sound and Vibration, pp 22-27, July 2000.

${ }^{4}$ Park, J. and Palumbo, D. L., "Measurements of Acoustic Properties of Porous and Granular Materials and Application to Vibration Control", Proc. Noise-CON 2004, Baltimore, MD, July 12-14, 2004.

${ }^{5}$ Song, B. H., and Bolton, J. S., "A Transfer-Matrix Approach for estimating the Characteristic Impedance and Wave Numbers of Limp and Rigid Porous Materials”, Journal of the Acoustic Society of America, Vol. 107, No. 3, 2000, pp. 1131-1152.

${ }^{6}$ Palumbo, D. L., and Park, J., "Improvements to the Two-Thickness Method for Deriving Acoustic Properties of Materials", Noise-Con 2004, Baltimore, MD, July 12-24, 2004.

${ }^{7}$ Palumbo, D.L.,"Evaluation of Acoustic Properties of Lightweight, Granular Materials", 9th AIAA/CEAS Conference and Exhibit, Hilton Head, So. Carolina, May 12-14, 2003.

${ }^{8}$ Fahy, F.J., "Sound and Structural Vibration”, Academic Press, p. 153, 1985.

${ }^{9}$ Biot, M. A., "Generalized Theory of Acoustic Propagation in Porous Dissipative Media", Journal of the Acoustical Society of America, Vol. 34, No. 9, 1962, pp. 1254-1262.

${ }^{10}$ Park, J., "Transfer Function Methods to Measure Dynamic Mechanical Properties of Honeycomb Structures and Beams", Journal of Sound and Vibration (to be published). 